\title{
Tendencia de la mortalidad por picaduras de alacrán en México, 1979-2003
}

\author{
Alfredo Celis, ${ }^{1}$ Ramón Gaxiola-Robles, ${ }^{2}$ Elizabeth Sevilla-Godínez, ${ }^{3}$ \\ María de Jesús Orozco Valerio ${ }^{4}$ y Jesús Armas $^{5}$
}

Forma de citar

Celis A, Gaxiola-Robles R, Sevilla-Godínez E, Orozco Valerio MJ, Armas J. Tendencia de la mortalidad por picaduras de alacrán en México, 1979-2003. Rev Panam Salud Publica. 2007;21(6):373-80.

RESUMEN Objetivo. Describir la tendencia de la mortalidad por picaduras de alacrán en México y sus estados federativos en el periodo de 1979 a 2003.

Métodos. Se estimaron las tasas crudas y estandarizadas de mortalidad por picaduras de alacrán y las tendencias durante el período estudiado a partir de los datos oficiales de mortalidad en México, según la 9. ${ }^{a}$ y la 10. ${ }^{a}$ revisiones de la Clasificación Internacional de Enfermedades (códigos E905.2 y X22, respectivamente). Los resultados se estratificaron por grupos de edad. Las frecuencias de muertes por picaduras de alacrán se compararon mediante el riesgo relativo (RR) y sus intervalos de confianza de 95\% (IC95\%).

Resultados. Entre 1979 y 2003, en México se registraron 6077 muertes por picaduras de alacrán. Se observó una tendencia descendente estadísticamente significativa en la mortalidad estandarizada $(\beta=-0,195 ; \mathrm{P} \leq 0,001)$, con una reducción total de $86,5 \%$ entre los períodos 1979-1982 y 2001-2003. La mayor mortalidad en el trienio 2001-2003 se observó en varones (0,81 por 1000000 de habitantes) y en los menores de 1 año (7,07 por 1000000 de habitantes), seguidos por los grupos de 1 a 4 años (3,78 por 1000000 de habitantes) y de 60 años o más (0,84 por 1000000 de habitantes). Las poblaciones con menos de 2500 habitantes presentaron una mortalidad por picaduras de alacrán superior en 11,8 (IC95\%: 7,86 a 17,72) veces la observada en las de más de 20000 habitantes. Los estados con mayor mortalidad por picaduras de alacrán se encuentran en la región occidental y central del país.

Conclusiones. A pesar de la sostenida tendencia descendente en el número de muertes por picaduras de alacrán en los últimos 20 años en México, este sigue siendo un importante problema de salud pública. Los grupos de edad más afectados son los niños menores de 5 años y los ancianos. Se deben tomar medidas para que en todas las comunidades, principalmente en las pequeñas, se disponga de los recursos y la información adecuados para la atención oportuna de las personas que sufren una picadura de alacrán.

Palabras clave Envenenamiento, escorpiones, mortalidad, México.

\footnotetext{
Departamento de Salud Pública, Universidad de Guadalajara y Unidad de Investigación Médica en Epidemiología Clínica, Hospital de Especialidades del IMSS, Guadalajara, Jalisco, México. La correspondencia se debe dirigir a Alfredo Celis, Universidad de Guadalajara, Departamento de Salud Pública, Sierra Morena No. 950, Edificio N, Colonia Independencia, Guadalajara, Jalisco CP44340, México. Correo electrónico: alfredo_celis@yahoo.com

2 Hospital General de Zona, Unidad de Medicina Familiar No. 1 del IMSS, La Paz, Baja California Sur, México.
}

Residencia de Epidemiología, Hospital de Pediatría del IMSS, Jalisco, México.

4 Departamento de Desarrollo Social, Universidad de Guadalajara, Guadalajara, Jalisco, México. (Actualmente en: Departamento de Salud Pública, Universidad de Guadalajara, Guadalajara, Jalisco, México.)

5 Instituto Nacional de Estadística, Geografía e Informática, Delegación de Jalisco, Guadalajara, Jalisco, México.
Entre las causas de muerte por reacciones tóxicas y envenenamientos ocasionados por plantas y animales venenosos, las picaduras de alacrán han ocupado el primer lugar en México durante más de 20 años (1). Ya desde 1996, Calderón-Aranda y colaboradores consideraban que México, con 
aproximadamente 250000 ataques al año, era el país con mayor incidencia de picaduras de alacrán (2). Datos actuales muestran que ese número no ha cambiado mucho: según el Boletín Nacional de Vigilancia Epidemiológica, en 2005 se registraron 247796 intoxicaciones por veneno de escorpión, cifra que aumentó hasta 269149 en 2006 (3). En México habitan aproximadamente 130 especies y subespecies de alacranes -todas pertenecientes al género Centruroides, familia Buthidae-, pero solo siete son de importancia médica: C. limpidus limpidus, C. limpidus tecomanus, C. infamatus, C. suffusus, C. noxius, C. elegans y C. sculpturatus. En México, todas estas especies y subespecies se localizan en las regiones occidental y central del país $(2,4)$.

El desenlace clínico del envenenamiento por la picadura de alacrán depende de múltiples factores, algunos propios del artrópodo y otros de la víctima. Entre los factores asociados con el alacrán se encuentran la especie de alacrán, las condiciones del telson (porción distal de la cola donde se encuentran las glándulas que inoculan el veneno), el número de picaduras y la cantidad de veneno inoculado. Los asociados con la víctima son la edad, el peso, las condiciones de salud y las enfermedades presentes (hipertensión arterial, enfermedad coronaria y diabetes, entre otras), así como el acceso a un tratamiento oportuno. El tiempo de aparición de los primeros signos y síntomas de envenenamiento y del desarrollo de reacciones graves puede ser muy corto y en la mayoría de los casos puede variar entre 5 y 30 minutos (2). El veneno del alacrán contiene una mezcla de polipéptidos tóxicos que afectan los canales celulares excitables y no excitables. Las familias de toxinas identificadas en el veneno de estos artrópodos actúan directamente sobre el organismo humano mediante el bloqueo o la disminución de la actividad de los canales de sodio, potasio y calcio. Al penetrar en el torrente sanguíneo, estas endotoxinas activan a los macrófagos a través de un receptor específico de membrana (CD14). Esto, a su vez, induce la cascada de la inflamación y genera una respuesta infla- matoria sistémica que lleva al shock séptico, la falla de múltiples órganos y la muerte (5).

A pesar de que las intoxicaciones por picaduras de alacrán constituyen un problema de salud pública en muchos países de África, Asia y las Américas, poco se sabe de los factores de riesgo asociados con este problema. Llama la atención que una búsqueda realizada en MEDLINE utilizando las palabras "scorpion" y "case control" o "cohort" no mostró estudios analíticos que describieran los factores de riesgo asociados con las intoxicaciones por picaduras de alacrán. Según Chowell y colaboradores (6), el número de ataques de alacrán es menor cuando la temperatura ambiente se encuentra por debajo de $16{ }^{\circ} \mathrm{C}$ y se incrementa a medida que aumenta la temperatura hasta alcanzar un máximo sostenido a alrededor de $19{ }^{\circ} \mathrm{C}$. Según algunos estudios descriptivos, la mayor cantidad de ataques se presenta durante la noche, en los menores de 15 años y en el hogar (7-9).

A pesar de su importancia como problema de salud pública, en México no se han estudiado los aspectos epidemiológicos de las intoxicaciones mortales por este artrópodo. El objetivo del presente trabajo es describir la tendencia de la mortalidad por picaduras de alacrán en México y sus estados federativos durante el período de 1979 a 2003.

\section{MATERIALES Y MÉTODOS}

Para esta investigación se utilizaron los datos oficiales de mortalidad en México correspondientes a los años de 1979 a 2003, tomados de las bases de datos del Instituto Nacional de Geografía, Estadística e Informática (INEGI). Esta información se puede consultar en el sitio de Internet del Sistema Nacional de Información (SINAIS) de México (10).

Para el análisis se tomaron en cuenta solamente los registros con diagnóstico de envenenamiento o reacciones tóxicas causadas por picaduras de alacrán, según los códigos E905.2 de la 9. ${ }^{a}$ revisión de la Clasificación Internacional de
Enfermedades (CIE-9) (11) y el código X22 de la 10. a revisión (CIE-10) (12).

Las variables analizadas fueron la edad y el sexo, así como el tamaño de la localidad, el estado federativo y el año en que se registró el suceso. La mortalidad se agrupó en trienios desde 1983 hasta 2003 y un cuatrienio inicial de 1979 a 1982.

La mortalidad por picaduras de alacrán se calculó como el número total de muertes por esa causa ocurridas en un período dado dividido entre el número de personas-año en el período. Para el análisis de la mortalidad se utilizaron tasas crudas y estandarizadas por la población (13), según el Censo General de Población y Vivienda del año 2000 (14), agrupada por edades en: menores de 1 año, de 1 a 4 , de 5 a 9 , de 10 a 19 , de 20 a 39, de 40 a 59 y 60 años o más.

El denominador de las tasas se proyectó linealmente (15) a partir de la población registrada en los Censos Generales de Población de 1980 (16), 1990 (17) y 2000 (14), y en el Conteo General de Población de 1995 (18). Para comparar la frecuencia de muertes por picaduras de alacrán se utilizó el riesgo relativo (RR) y sus intervalos de confianza de 95\% (IC95\%). Las tendencias se analizaron mediante regresión simple y múltiple, según correspondiera. El nivel de significación fue de 0,05. La descripción gráfica de la mortalidad por estado federativo se realizó mediante el paquete estadístico Epi Map, de Epi Info para Windows (19).

\section{RESULTADOS}

Entre 1979 y 2003, en México se registraron 6077 muertes por picaduras de alacrán. Se observó una tendencia descendente estadísticamente significativa en la mortalidad estandarizada $(\beta=-0,195 ; P \leq 0,001)$ (figura 1$)$. Entre los períodos 1979-1982 y 2001-2003, la reducción en la mortalidad por picaduras de alacrán en el país fue de $86,5 \%$. También se constataron reducciones en los estados del país que registraron defunciones por esta causa en el período 1979-1982 y, aunque se observaron incrementos puntuales en 
FIGURA 1. Tendencia de la mortalidad estandarizada ${ }^{a}$ por picaduras de alacrán. México, 1979-2003

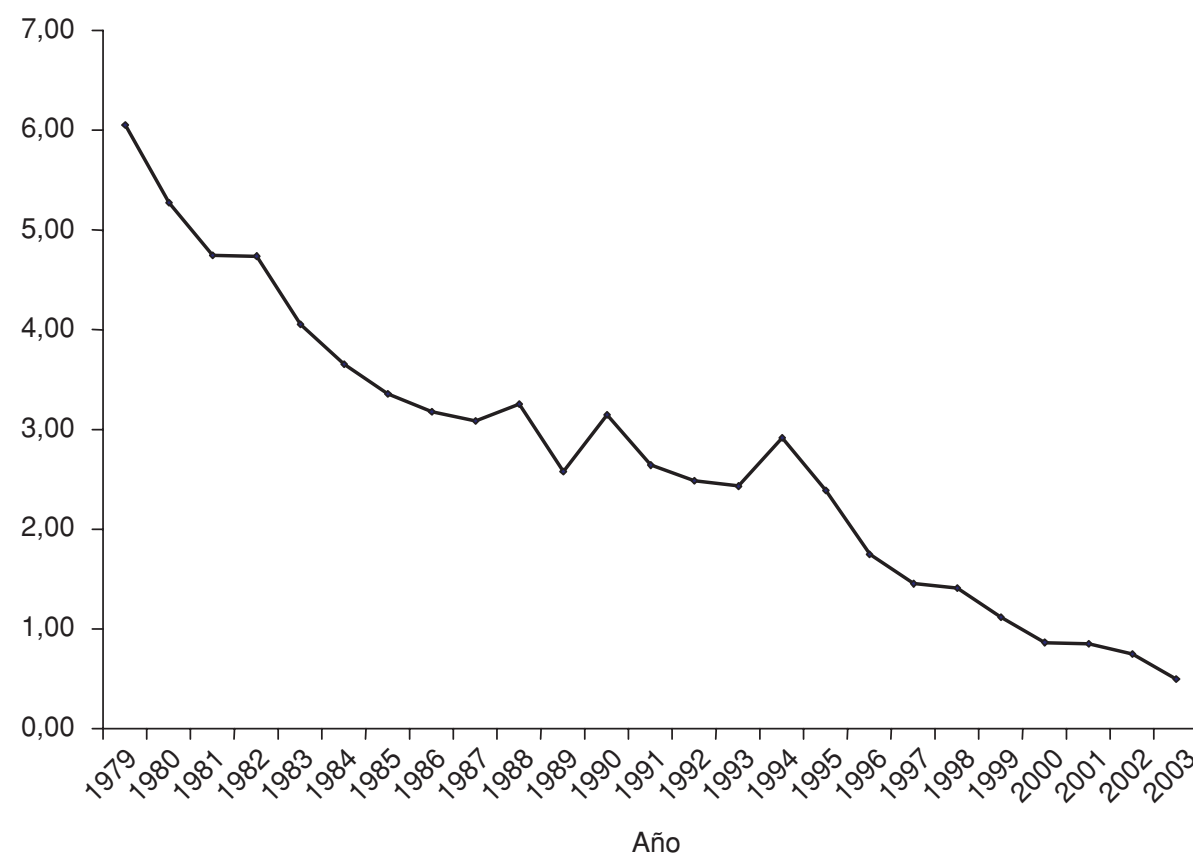

Fuente: Elaborado a partir del análisis de los certificados de defunción de la Secretaría de Salud, México (10). a Por 1000000 de habitantes, según la población de México en el año 2000 (5).

algunos años, la tendencia general entre 1979 y 2003 fue de disminución en el número de muertes (cuadro 1). En el período 2001-2003 se registraron 206 defunciones por picaduras de alacrán. Un caso particularmente notable es el del estado de Colima, que pasó de tener la mayor mortalidad estandarizada en el trienio 1979-1982 (53,8 por 1000000 de habitantes) a no presentar muertes por esta causa en el trienio 2001-2003.

En la figura 2 se ilustra la distribución de la mortalidad por picaduras de alacrán en los estados federativos de México en el trienio 2001-2003. En ella se observa que los estados con mayor mortalidad por picaduras de alacrán en ese trienio se encuentran en la región occidental y central del país. En rasgos generales, este patrón en la distribución geográfica se mantuvo durante todo el período estudiado (cuadro 1) y el número de defunciones notificadas por esta causa en otros estados mexicanos en años anteriores fue siempre muy reducido.
Según los grupos de edad, la mayor mortalidad en el trienio 2001-2003 se observó en niños menores de 1 año (7,07 por 1000000 de habitantes), seguidos por el grupo de 1 a 4 años $(3,78$ por 1000000 de habitantes) y el de 60 años o más (0,84 por 1000000 de habitantes) (cuadro 2). En general, los eventos mortales ocurrieron con mayor frecuencia en varones, aunque la diferencia con respecto a las mujeres y niñas no alcanzó el nivel de significación estadística establecido $(P=0,051)$.

La distribución de la mortalidad por picaduras de alacrán según el tamaño de la localidad demostró que las poblaciones con menos de 2500 habitantes presentaron una tasa superior en 11,8 (IC95\%: 7,86 a 17,72) veces a la observada en las localidades con más de 20000 habitantes (cuadro 3). En la figura 3 se puede observar que si bien la tendencia de la mortalidad por picaduras de alacrán disminuyó significativamente en todas las categorías $(P<0,01)$, esa reducción fue significativamente mayor en las poblaciones con menos de 20000 habitantes que en las poblaciones con una población mayor $(P<0,01)$.

\section{DISCUSIÓN}

Las muertes por picaduras de alacrán constituyen un grave problema de salud en México y son más frecuentes en la población infantil. Como se ha demostrado en este estudio, $68,4 \%$ de las muertes notificadas corresponden a niños menores de 5 años de edad. A partir de esa edad, la tasa de mortalidad disminuye y vuelve a aumentar a partir del grupo de 40-59 años. Estos resultados concuerdan con lo informado por otros autores $(1,20,21)$, que destacan la importancia de esta causa de muerte en menores de 15 años. Díaz-Dueñas y Tene (22) y Al-Asmari y Al-Saif (23) también observaron un aumento del riesgo en edades tempranas y en los adultos mayores.

La vulnerabilidad a las picaduras de alacrán en las edades tempranas puede deberse a que los niños tienen menor índice de masa corporal y, por lo tanto, la cantidad de veneno inoculado resulta letal en una mayor proporción de casos (24). La independencia y el deseo de explorar de los niños y niñas de entre 2 y 6 años de edad los expone a un mayor riesgo de contacto con animales ponzoñosos, como los alacranes (1). Por otra parte, el incremento de las tasas de mortalidad observada en los muy pequeños y en las personas mayores de 60 años podría explicarse por el hecho de que pasan más tiempo en el hogar y es ahí donde ocurren más de $90 \%$ de las picaduras de alacrán (9). Ramsey y colaboradores (25) informaron haber visto alacranes en $12-18 \%$ de las viviendas de Chalcatzingo, Morelos, uno de los estados de México con mayor número de muertes registradas por picaduras de alacrán.

La distribución geográfica de las defunciones registradas en 2001-2003 muestra un patrón bien definido, con frecuencias más elevadas en la mayoría de los estados federativos que tienen costas en el Océano Pacífico (Sonora, Sinaloa, Nayarit, Jalisco, Michoa- 
CUADRO 1. Mortalidad estandarizada a por picaduras de alacrán según el estado federativo. México, 1979-2003

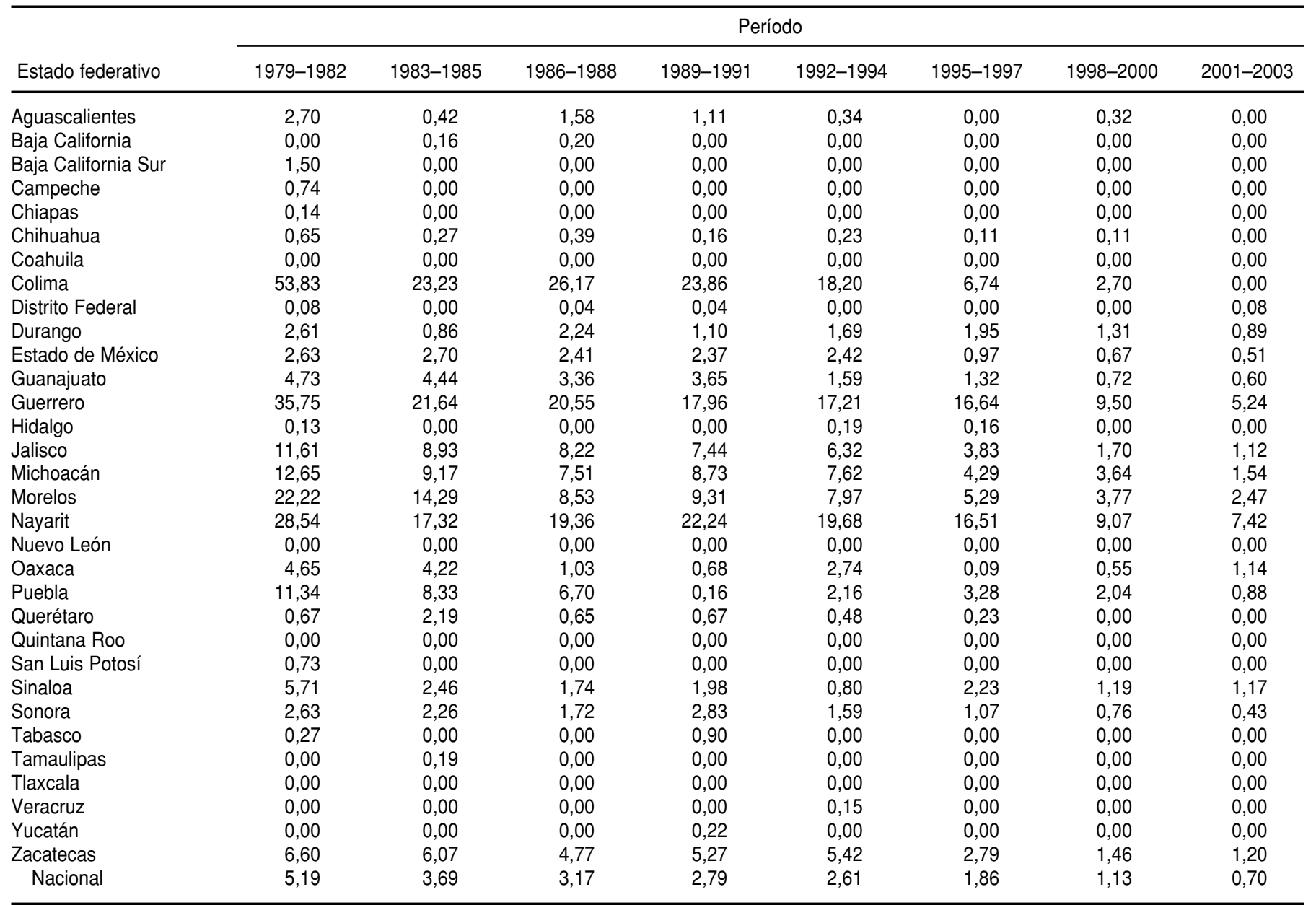

Fuente: Elaborado a partir del análisis de los certificados de defunción de la Secretaría de Salud, México (10).

a Por 1000000 habitantes, según la población de México en el año 2000 (5).

cán, Guerrero y Oaxaca) y en algunos estados de la región central (Durango, Zacatecas, Guanajuato, Estado de México, Puebla y Morelos). Este comportamiento puede deberse a que los tipos de alacranes que habitan en estas regiones son los más tóxicos del país $(4,26)$. Por ejemplo, C. noxius (la especie de alacrán más letal para las personas) se localiza en la mayor parte del estado de Nayarit, donde la mortalidad estandarizada por esta causa se ha mantenido muy elevada a lo largo de todo el período estudiado. Esta especie se encuentra también en los estados colindantes, aunque en menor medida. Otras especies venenosas encontradas son C. suffusus, en Durango y Sinaloa; C. limpidus limpidus, en More- los y Guerrero; C. limpidus tecomanus, en Colima y Jalisco; y C. elegans en Nayarit, Jalisco, Michoacán, Guerrero y Oaxaca. Estas especies se encuentran con muy poca frecuencia en los estados adyacentes con el Golfo de México o en otros estados del norte y el centro del país, como Chihuahua, Coahuila, Nuevo León, San Luis Potosí, Aguascalientes, Querétaro, Hidalgo, Tlaxcala y el Distrito Federal. Además, no se han encontrado en la Península de Yucatán $(2,26)$.

Los resultados de esta investigación indican que el tamaño de la población está asociado con la mortalidad por picaduras de alacrán: cuanta más pequeña es la localidad, mayor es el riesgo de morir por la picadura de ala- crán. Esto puede deberse a diversas causas: primero, al menor acceso a los servicios de salud en las zonas rurales y marginadas - formadas por lo general por comunidades pequeñas-, al mayor tiempo necesario para recorrer la distancia que separa a esas comunidades de los servicios de salud, a los inconvenientes para encontrar un transporte adecuado y oportuno y a la carencia de caminos apropiados (27); segundo, la existencia de prácticas y creencias mágico-religiosas y de una extensa gama de remedios de origen herbolario, animal o de otra naturaleza, aplicados por brujos, chamanes o curanderos, tiende a retardar la atención oportuna de los pacientes por personal calificado $(1,28,29)$. 
FIGURA 2. Mortalidad estandarizada ${ }^{a}$ por picaduras de alacrán según el estado federativo. México, 2001-2003

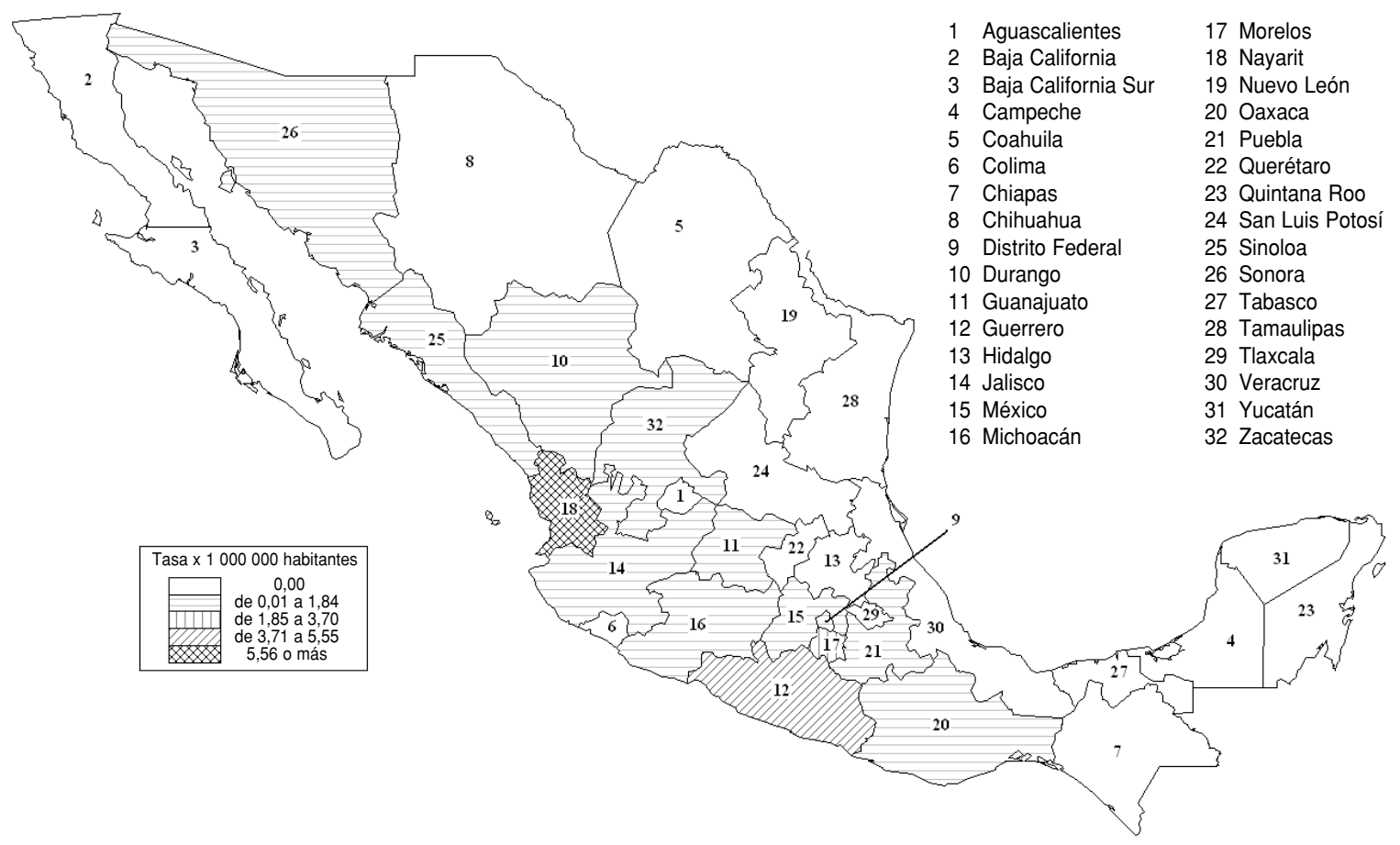

Fuente: Elaborado a partir del análisis de los certificados de defunción de la Secretaría de Salud, México (10). a Por 1000000 de habitantes, según la población de México en el año 2000 (5).

CUADRO 2. Tasa de mortalidad ${ }^{\mathrm{a}}$ por picaduras de alacrán por millón de habitantes, según el grupo de edad y el sexo. México, 2001-2003

\begin{tabular}{|c|c|c|c|c|c|c|c|c|c|}
\hline \multirow{2}{*}{$\begin{array}{l}\text { Grupo de } \\
\text { edad, años }\end{array}$} & \multicolumn{3}{|c|}{ Hombres y niños } & \multicolumn{3}{|c|}{ Mujeres y niñas } & \multicolumn{3}{|c|}{ Total } \\
\hline & No. & Tasa $^{a}$ & IC95\% ${ }^{b}$ & No. & Tasa $^{a}$ & IC95\% ${ }^{b}$ & No. & Tasa $^{a}$ & IC95\% ${ }^{b}$ \\
\hline Menos de 1 & 25 & 7,86 & 4,96 a 10,94 & 19 & 6,18 & 3,40 a 8,96 & 44 & 7,07 & 4,98 a 9,16 \\
\hline De 1 a 4 & 50 & 3,86 & 2,79 a 4,92 & 47 & 3,42 & 2,44 a 4,40 & 97 & 3,78 & 3,03 a 4,54 \\
\hline De 5 a 9 & 12 & 0,70 & 0,30 a 1,09 & 9 & 0,53 & 0,18 a 0,88 & 21 & 0,62 & 0,36 a 0,89 \\
\hline De 10 a 19 & 5 & 0,16 & 0,02 a 0,30 & 1 & 0,03 & 0,00 a 0,10 & 6 & 0,10 & 0,02 a 0,17 \\
\hline De 20 a 39 & 3 & 0,07 & 0,00 a 0,14 & 4 & 0,08 & 0,00 a 1,16 & 7 & 0,08 & 0,02 a 0,13 \\
\hline De 40 a 59 & 7 & 0,30 & 0,08 a 0,53 & 6 & 0,24 & 0,05 a 0,43 & 13 & 0,28 & 0,13 a 0,43 \\
\hline 60 o más & 13 & 1,26 & 0,58 a 1,95 & 5 & 0,47 & 0,06 a 0,89 & 18 & 0,84 & 0,45 a 1,23 \\
\hline Total & 115 & 0,81 & 0,66 a 0,96 & 91 & 0,61 & 0,48 a 0,73 & 206 & 0,71 & 0,62 a 0,81 \\
\hline
\end{tabular}

Fuente: Elaborado a partir del análisis de los certificados de defunción de la Secretaría de Salud, México (10).

a Tasa por 100000 de habitantes.

b IC95\%: intervalo de confianza de $95 \%$.

En los últimos 24 años, la mortalidad por picaduras de alacrán ha mostrado una pronunciada tendencia descendente. Esta disminución se explica por el desarrollo social, económico y médico-tecnológico que se ha evidenciado en México. Aunque no se puede cuantificar el efecto de cada uno de los factores que influyeron en la disminución de la mortalidad por picaduras de alacrán, el mejoramiento de las condiciones de vida, vivienda, higiene pública, transporte y comunicaciones y la aplicación de programas eficaces de salud pública, así como la mayor accesibilidad a los servicios de salud han debido influir directamente en esa reducción (27). Según investigaciones realizadas en Marruecos, los que reciben atención en los servicios de salud tienen una mayor probabilidad de so- 
CUADRO 3. Tasa de mortalidad por picaduras de alacrán por millón de habitantes, según el tamaño de la localidad. México, 2001-2003

\begin{tabular}{lrcrc}
\hline $\begin{array}{c}\text { Tamaño de la localidad, } \\
\text { habitantes }\end{array}$ & No. & $\begin{array}{c}\text { Tasa por } \\
1000000 \text { de } \\
\text { habitantes }\end{array}$ & RR $^{\mathrm{a}}$ & IC95\% $^{\mathrm{b}}$ \\
\hline De 1 a 2 499 & 138 & 1,84 & 11,80 & 7,86 a 17,72 \\
De 2 500 a 19 999 & 37 & 0,79 & 5,05 & 3,09 a 8,26 \\
20 000 o más & 28 & 0,16 & 1,00 & \\
\hline
\end{tabular}

Fuente: Elaborado a partir del análisis de los certificados de defunción de la Secretaría de Salud, México (10). ${ }^{\mathrm{a}} \mathrm{RR}$ : riesgo relativo.

bIC95\%: intervalo de confianza de $95 \%$.

FIGURA 3. Tendencia de la mortalidad por picaduras de alacrán según el tamaño de la localidad. México, 1979-2003

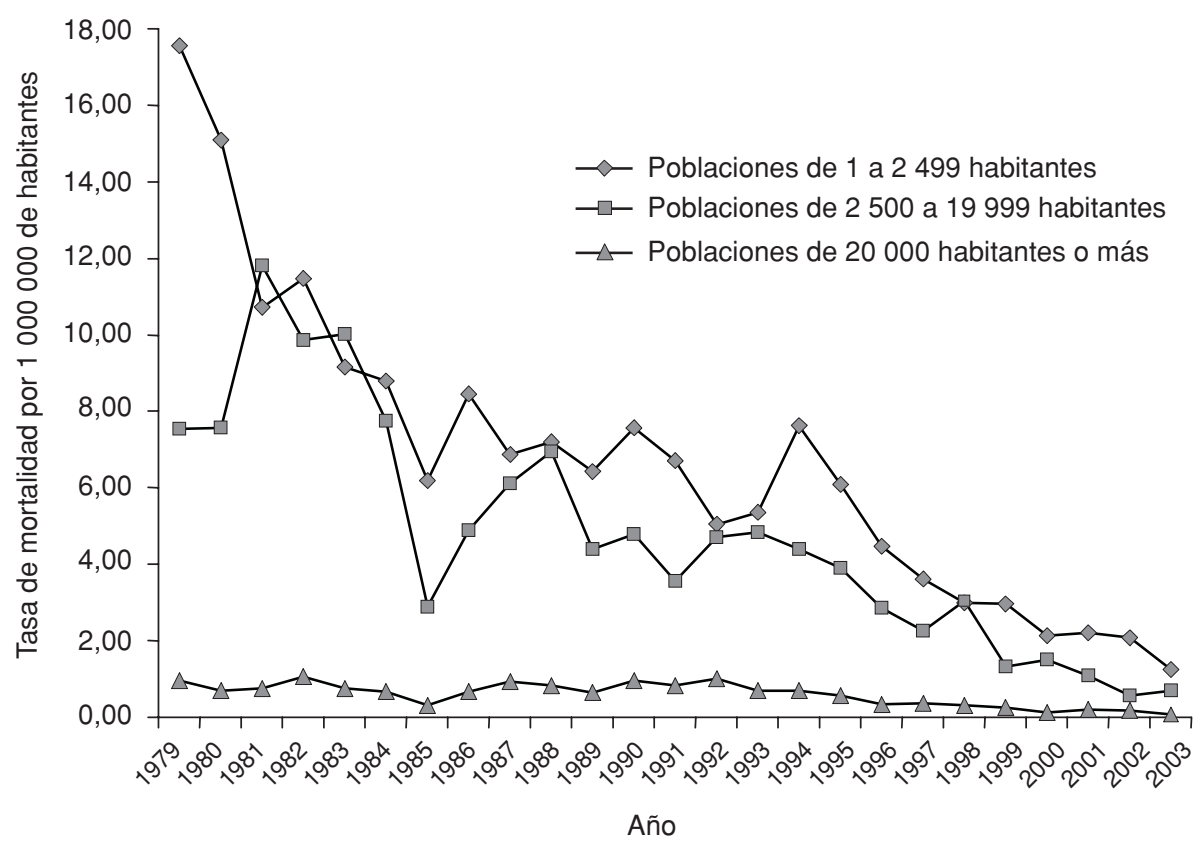

Fuente: Elaborado a partir del análisis de los certificados de defunción de la Secretaría de Salud, México (10).

brevivir a una picadura de alacrán que quienes no la reciben (7), por lo que el acceso oportuno a los servicios de salud y la calidad de los mismos son factores determinantes para salvar la vida de las personas afectadas.

La utilidad de los antisueros contra el veneno de alacrán es un tema controversial. Si bien Abroug y colaboradores (30) no encontraron beneficios en su uso al compararlo con un placebo en una muestra de 825 pacientes mayores de 10 años en Túnez, otros autores informaron resultados satisfactorios al utilizarlo $(2,31,32)$. Gracias a la disponibilidad de estos antisueros y a su amplia distribución en muchas comunidades, en Jalisco se ha logrado reducir notablemente la mortalidad por picaduras de alacrán (33).

Si bien la drástica reducción de la mortalidad observada en el estado de Colima se podría atribuir a omisiones en la notificación, la tendencia observada en el cuadro 1 demuestra que esa disminución ha sido progresiva y constante a lo largo de todo el período analizado, lo que no respalda esa hipótesis. Es necesario realizar estudios específicos más detallados que permitan identificar los factores que han llevado a esta reducción en el estado de Colima.

En el caso del Distrito Federal, no se registraron muertes entre 1991 y 2001. En 2002 se registró la muerte de dos personas residentes en ese estado, pero esas muertes se registraron fuera del Distrito Federal. Dada la urgencia que representan estos eventos, se considera que la atención médica (si la hubiera) y la muerte ocurren en el mismo estado en que ocurre el ataque del alacrán.

Otro caso interesante está relacionado con la baja mortalidad por picaduras de alacrán observada en el estado de Chiapas, ubicado en la zona de mayor mortalidad por esta causa y con una gran población rural. Esta aparente contradicción podría explicarse por el hecho de que en ese estado no habitan especies de alacranes de gran toxicidad (2).

Este estudio tiene limitaciones, ya que se basó en los registros de los certificados de defunción, lo que reduce el número de variables que se pueden analizar. Además, la validez de los resultados depende de la calidad de los registros y se sabe que en la mayoría de las poblaciones pequeñas se registra un elevado número de muertes sin causa determinada (34). Como en estas comunidades existe el mayor riesgo de morir por la picadura de alacrán, el efecto de este sesgo puede ser importante. Por otra parte, en este estudio no se tuvo en cuenta una arista importante del problema: la morbilidad. Las estadísticas oficiales mexicanas muestran que en los últimos siete años, el número de picaduras de alacrán informadas ha aumentado sistemáticamente de 206098 en 2000 a 269149 en $2006(3,35,36)$.

\section{CONCLUSIONES Y RECOMENDACIONES}

Los resultados de este trabajo demostraron la sostenida tendencia descen- 
dente en el número de muertes por picaduras de alacrán en los últimos 20 años en México. No obstante, este sigue siendo un importante problema de salud pública y los grupos de edad más afectados son los niños y los ancianos.

Se deben tomar medidas para que en todas las comunidades, ya sean grandes o pequeñas, se disponga de los recursos adecuados para la atención oportuna de las personas que sufren una picadura de alacrán. En los lugares distantes de los servicios de salud se debe contar con un plan para el traslado inmediato de esas personas a los servicios de atención médica. Se debe brindar la información precisa de los lugares adonde se debe acudir para recibir atención médica en caso necesario.

Por último, es importante mejorar los sistemas de registro, particularmente en lo referente a los ataques no mortales.

\section{REFERENCIAS}

1. Hijar M, Blanco J, Carrillo C, Rascón A. Mortalidad por envenenamiento en niños. Salud Publica Mex. 1998;40(4):347-53.

2. Calderón-Aranda ES, Dehesa-Dávila M, Chávez-Haro A, Possani LD. Scorpion stings and their treatment in Mexico. En: Bon C, Goyffon M, eds. Envenomings and their treatments. Lyon: Fondation Marcel Merieux; 1996. Pp. 311-20.

3. Sistema Nacional de Vigilancia Epidemiológica. Epidemiología. Sistema único de Información. México: Secretaría de Salud; 2007. Hallado en http://www.dgepi.salud.gob. $\mathrm{mx} /$ boletin/2007/sem01/pdf/cua11.4.pdf. Acceso el 12 de julio de 2007.

4. Montoya-Cabrera MA. Alacranismo. Gac Med Mex. 1996;132:645-8.

5. Scharf S. Scorpion sting-the inflammatory dimension. Crit Care Med. 2002;30 (7): 1669-70

6. Chowell G, Hyman JM, Díaz-Dueñas P, Hengartner NW. Predicting scorpion sting incidence in an endemic region using climatological variables. Int J Environ Health Res. 2005; 15(5):425-35.

7. Soulaymani-Bencheikh R, Soulaymani A, Semlali I, Tamim OK, Zemrour E, Eloufir R, et al. Les piqûres et les envenimations scorpioniques au niveau de la population de Kouribga (Maroc). Bull Soc Pathol Exot. 2005;98(1): 36-40.

8. Otero R, Navío E, Céspedes FA, Núñez MJ, Lozano L, Moscoso ER, et al. Scorpion envenoming in two regions of Colombia: clinical, epidemiological and therapeutic aspects. Trans $\mathrm{R}$ Soc Trop Med Hyg. 2004;98(12):742-50.

9. De Armoni AM, Carvalho FM, Lira-da-Silva RM, Brazil TK. Acidentes por escorpao em uma area do nordeste de Amaralina, Salvador, Bahia, Brasil. Rev Soc Bras Med Trop. 2003;36(1):51-6.

10. Sistema Nacional de Información. Mortalidad. México, D.F.: Secretaría de Salud; 2007. Hallado en http://sinais.salud.gob.mx/ mortalidad/. Acceso el 17 de julio de 2007.

11. Organización Mundial de la Salud. Clasificación estadística internacional de enfermedades, traumatismos y causas de defunción. 9. ${ }^{\text {a }}$ revisión. Washington, D.C.: Organización Panamericana de la Salud; 1978.

12. Organización Mundial de la Salud. Clasificación estadística internacional de enfermedades y problemas relacionados con la salud. 10. ${ }^{a}$ revisión. Washington, D.C.: Organización Panamericana de la Salud; 1998.
13. Rotman K, Greenland S. Moderm epidemiology. Philadelphia: Lippincott-Raven; 1998.

14. Instituto Nacional de Estadísticas, Geografía e Informática. Censo General de Población y Vivienda, 2000 [base de datos en CD]. Aguascalientes, México: INEGI; 2001.

15. Camel F. Estadística médica y planificación de la salud. Vol II. Mérida, Venezuela: Consejo de Publicaciones de la Universidad de Los Andes; 1982

16. Instituto Nacional de Estadísticas, Geografía e Informática. Censo General de Población y Vivienda, 1980. México, D.F.: INEGI; 1984.

17. Instituto Nacional de Estadísticas, Geografía e Informática. Resumen general. XI Censo General de Población y Vivienda. Aguascalientes: INEGI; 1992

18. Instituto Nacional de Estadísticas, Geografía e Informática. Conteo de Población y Vivienda 1995. Resultados definitivos. Tabulados básicos. Aguascalientes: INEGI; 1997.

19. Dean AG, Arner TG, Sangam S, Sunki GG, Friedman R, Lantinga M, et al. Epi Info 2000, a database and statistics program for public health professionals for use on Windows 95 , 98, NT, and 2000 computers. Atlanta, Georgia: Centers for Disease Control and Prevention; 2000.

20. Soulaymani-Bencheikh R, Semlali I, Ghani A, Badri M, Soulaymani A. Establishment and analysis of a log to record scorpion stings in Morocco. Sante Publique. 2004;16(3):487-98.

21. Chowell G, Díaz-Dueñas P, Bustos-Saldana R, Mireles AA, Fet V. Epidemiological and clinical characteristics of scorpionism in Colima, Mexico (2000-2001). Toxicon. 2006;47(7):753-8.

22. Díaz-Dueñas $P$, Tene CE. Alacranismo en Colima, aspectos epidemiológicos. Rev Med IMSS. 2001;39;517-21.

23. Al-Asmari AK, Al-Saif AA. Scorpion sting syndrome in a general hospital in Saudi Arabia. Saudi Med J. 2004;25(1):64-70.

24. Nunan EA, Moraes M, Cardoso V, MoraesSantos T. Effect of age on body distribution of tityustoxin from Tityus serrulatus scorpion venom in rats. Life Sci. 2003;73(3):319-25.

25. Ramsey JM, Salgado L, Cruz-Celis A, López R, Alvear L, Espinosa L. Domestic scorpion control with pyrethroid insecticides in Mexico. Med Vet Entomol. 2002;16(4):356-63.

26. Pinkus-Rendón MA. Alacranes sinantrópicos de Mérida, Yucatán, México. Rev Biomedica. 1999;10:153-8.

27. López-Ríos O. Efectos de los servicios de salud y de factores socioeconómicos en las di- ferencias espaciales de la mortalidad mexicana. Salud Publica Mex. 1997;39(1):16-24.

28. Aguilar PR, Ramírez GS, Villegas SE. Conceptos populares acerca de la picadura de alacrán. Gac Med Mex. 1979;115;243-4.

29. Aldana GB, Aldana GMR. Alacranismo en Jalisco: estudio de 115 pacientes atendidos en consultorio. Rev Med IMSS. 1992;30:109-13.

30. Abroug F, ElAtrous S, Nouira S, Haguiga H, Touzi N, Bouchoucha S. Serotherapy in scorpion envenomation: a randomised controlled trial. Lancet. 1999;354(9182):906-9.

31. Osnaya-Romero N, Medina-Hernández TJ, Flores-Hernández SS, León-Rojas G. Clinical symptoms observed in children envenomated by scorpion stings, at the children's hospital from the State of Morelos, Mexico. Toxicon. 2001;39:781-5.

32. LoVecchio F, McBride C. Scorpion envenomations in young children in central Arizona. J Toxicol Clin Toxicol. 2003;41(7):937-40.

33. Estados Unidos Mexicanos, Secretaría de Salud de Jalisco. Programa de prevención y control de la picadura de alacrán. Guadalajara: Secretaría de Salud y Bienestar de Jalisco; 2005.

34. Miranda-Ocampo R, Salvatierra IB, VivancoCedeño B, Álvarez-Lucas C, Lezana-Fernández M. Inequidad de los servicios de salud a población abierta en México. Salud Publica Mex. 1993;35(6);576-84.

35. Sistema Nacional de Vigilancia Epidemiológica. Epidemiología. Sistema único de Información. México: Secretaría de Salud; 2007. Hallado en http://www.dgepi.salud.gob. $\mathrm{mx} /$ boletin/2002/sem1/cua11.pdf. Acceso el 12 de julio de 2007.

36. Sistema Nacional de Vigilancia Epidemiológica. Epidemiología. Sistema único de información. México: Secretaría de Salud; 2007. Hallado en http://www.dgepi.salud.gob. $\mathrm{mx} /$ boletin/2004/sem01/pdf/cua11.1.pdf. Acceso el 12 de julio de 2007.

Manuscrito recibido el 11 de julio de 2006. Aceptado para publicación, tras revisión, el 4 de junio de 2007. 


\section{ABSTRACT \\ Trends in mortality from scorpion stings in Mexico, 1979-2003}

Key words Scorpions, bites and stings, mortality, risk factors, Mexico. whole and in each of its states for the period of 1979 to 2003. risk (RR), with $95 \%$ confidence intervals (CIs). regions of the country. scorpion sting.

Objective. To describe the trends in mortality from scorpion stings in Mexico as a

Methods. We estimated the crude and standardized mortality rates due to scorpion stings and the trends during the period studied based on official mortality data for Mexico, using the codes (E905.2 and X22, respectively) from the 9th and 10th editions of the International Classification of Diseases. The results were stratified by age group. The frequencies of deaths from scorpion stings were compared using relative

Results. Between 1979 and 2003 in Mexico, 6077 deaths from scorpion stings were registered. A statistically significant downward trend was found in standardized mortality rates $(\beta=-0.195 ; P \leq 0.001)$, with a total reduction of $86.5 \%$ for the period of 2001-2003 versus 1979-1982. For the 2001-2003 period, the highest mortality rates were in children under 1 year of age ( 7.07 per 1000000$)$, children 1 to 4 years old (3.78 per 1000000$)$, persons 60 and older (0.84 per 1000000$)$, and males (0.81 per 1000000$)$. Persons in communities with fewer than 2500 inhabitants had a relative risk that was 11.8 times (95\% CI: 7.86 to 17.72) that found in communities with more than 20000 inhabitants. The states with the highest mortality rates were in the central and western

Conclusions. Despite the sustained decline in the number of deaths from scorpion stings in the last 20 years in Mexico, there is still an important public health problem. The groups that are most affected are children under 5 and the elderly. Measures should be taken so that in all communities, especially small ones, adequate resources and information are available to provide for the prompt care of persons who suffer a

\section{Zoonosis y enfermedades transmisibles comunes al hombre y a los animales, $3^{\mathrm{a}}$ edición}

Este popular libro presentado en tres volúmenes, útil para las escuelas de salud pública, medicina, medicina veterinaria, organismos de salud pública y de salud animal, ofrece información sobre las zoonosis y las enfermedades transmisibles en América Latina y el resto del mundo. Desde su primera edición, en 1977, se han producido grandes adelantos científicos en relación con estas enfermedades, gracias a nuevas tecnologías y a los avances de la epidemiología, ecología y las demás ciencias biológicas y sociales, los cuales se han tomado en cuenta en esta tercera edición.

Volumen I: Bacteriosis y micosis

Pedro N. Acha y Boris Szyfres • 2001 • 416 pp. • ISBN 9275315809 • US\$ 36.00 en América Latina y el Caribe/ US $\$ 30.00$ en el resto del mundo •Código: PC 580 A Disponible en inglés: SP 580

Volumen II: Clamidiosis, rickettsiosis y virosis

Pedro N. Acha y Boris Szyfres • 2003 • 480 pp. • ISBN 9275319928 • US\$ 36.00 en América Latina y el Caribe/ US\$ 30.00 en el resto del mundo • Código: PC 580B • Disponible en inglés: SP 580B

Volumen III: Parasitosis

Pedro N. Acha y Boris Szyfres • $2003 \cdot 544$ pp. • ISBN $9275319936 \cdot$ US $\$ 36.00$ en América Latina y el Caribe/ US\$ 30.00 en el resto del mundo • Código: PC 580C • Disponible en inglés: SP $580 \mathrm{C}$

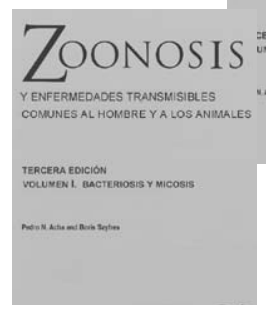

Usted también puede enviar su pedido a: PAHO Sales and Distribution Center P.O. Box 27 Annapolis Junction, MD 20701-0027 USA • http://publications.paho.org•Fax: (301) 206-9789•E-mail: paho@pmds.com 